\title{
Magnetism of $\mathrm{Co}_{13}$-Filled Carbon Nanotubes of Diverse Chiral Symmetry
}

\author{
Andrew Kuznetsov \\ ATG:biosynthetics, Merzhausen, Germany \\ Email: kuznet61@gmail.com
}

Received December 28, 2012; revised January 30, 2013; accepted February 9, 2013

\begin{abstract}
The attempt to study magnetism in $(n, m)$ chiral space of single-walled carbon nanotubes (SWNTs) with embedded metal cluster is presented. $\mathrm{Co}_{13}$ metallic cluster inside zigzag and chiral single-walled nanotubes was investigated using density functional theory (DFT). Magnetic properties of the endohedral nanotubes with the various chiral index $(n, m)$ were characterized by calculation of the total spin magnetic moment $(\mathrm{S})$. The dependence of $\mathrm{S}$ on the chiral symmetry of nanotubes, as well as the orientation of $\mathrm{Co}_{13}$ cluster within nanotubes was found. Longitudinal orientation of icosahedral $\mathrm{Co}_{13}$ cluster was preferable for magnetization in general. However, it was shown that the magnetic landscape $M=f(n, m)$ of endohedral nanotubes is very complex and sharp.
\end{abstract}

Keywords: Magic $\mathrm{Co}_{13}$ Cluster; Endohedral Nanotubes; Chirality; Total Spin Magnetic Moment

\section{Introduction}

Thorough investigation of nanoobjects, such as metal [1] and carbon [2] clusters, is essential because their characteristics are unlike bulk materials [3,4]. Carbon fullerenes and nanotubes have been paid immense attention to since they were discovered in 1985 and $1991[5,6]$. Tiny space inside a fullerene or a carbon nanotube (CNT) may be considered as a unique place for atomic clusters. The endohedral fullerenes and CNTs show many remarkable physical and chemical features [7-12].

I reported recently on computational results on magnetic properties of putative $\mathrm{Co}_{n} @ \mathrm{C}_{m}(n=5,13 ; m=60,70$ and 80 ) endohedral metallofullerenes [13,14]. Instead of fullerenes, single-walled nanotubes (SWNTs) may be preferable, because ends of nanotube are open allowing entering the tube [15-17]. In this paper, I use the same computational approach as done in $[13,14]$ to study the magnetism of SWNTs with various chirality which were filled by the icosahedral magnetic $\mathrm{Co}_{13}$ cluster in alternative orientations to the nanotube's channel.

\section{Method}

CoNTub 1.0 program $[18,19]$ was used to determine atomic coordinates of SWNTs with chiral vector $(n, m)$. The diameter of nanotubes was calculated from $(n, m)$ indices as follows:

$$
d=\frac{a}{\pi} \sqrt{\left(n^{2}+n m+m^{2}\right)},
$$

where $a=0.246 \mathrm{~nm}$. Obtained nanotubes were filled by $\mathrm{Co}_{13}$ cluster using SPDB viewer 4.1 [20]. Magnetic moments of the endohedral nanotubes were calculated on the basis of density functional theory (DFT) [21] within OpenMX 3.5 software [22-25]. Two density functionals, such as the local spin density approximation of CeperleyAlder (LSDA-CA) [26] or the Perdew-Burke-Ernzerhof generalized gradient approximation (GGA-PBE) [27], both with the active spin polarization, were used to compare outputs. Energy convergence was performed by the generalized divide-conquer optimization technique using parameters fitted to d-orbitals of Co-atoms. The self consisted field (SCF) criterion corresponded to $6 \times 10^{-6} \mathrm{Har}-$ tree. The atomic species were defined as C4.0-s1p1 and Co5.5-s2p2d2f1. Electronic temperature was $300.0 \mathrm{~K}$ and the energy cutoff was $200.0 \mathrm{Ry}$. Total spin S was accepted as a value of magnetic moment.

\section{Data Processing and Results}

The computation parameters for pure Co clusters were chosen and checked as described previously in $[13,14]$. The idea was to calculate metal-carbon complexes using the OpenMX's basis set, which was originally developed to simulate large systems [22]. I also used the relative 
assessment and internal reference. The values of magnetic moments for $\mathrm{Co}_{5}$ and $\mathrm{Co}_{13}$ clusters were obtained, such as 2.60 and $2.39 \mu_{\mathrm{B}}$ /atom respectively, which is in agreement with [28]. It is higher than for the bulk Co material, $1.62 \mu_{B}$ [29]. The computational procedure was tested on $\mathrm{Co}_{5}-$ and $\mathrm{Co}_{13}$-carbides giving reliable results. LDA and GGA approximations demonstrated similar outputs $\left(R_{L D A / G G A}=0.99\right)$. However, the standard deviation of magnetic moments for $\mathrm{Co}_{13} \mathrm{C}_{12}$ carbides was bigger than for $\mathrm{Co}_{5} \mathrm{C}_{5}$ carbides after five independent runs for each system, such that 0.0521 versus $0.0004 \mu_{\mathrm{B}}$ [13]. That allowed me to use this computational approach to investigate the endohedral fullerenes $\mathrm{Co}_{\mathrm{n}} @ \mathrm{C}_{\mathrm{m}}(n=5,13 ; m=$ $60,70,80)$, then to attack the endohedral nanotubes filled with $\mathrm{Co}_{13}$ cluster. The length of SWNTs was $10 \AA$; the number of carbon atoms varied around 60. For example, the zig-zag $(7,0)$ tube consisted of $56 \mathrm{C}$-atoms, as well as the chiral $(5,3)$ or $(6,2)$ tube included $64 \mathrm{C}$-atoms. As the gradient and sub-space optimization methods did not allow always reaching a ground state for complex systems, the SCF criterion was decreased to $10^{-6}$ Hartree.

In experiments, I varied the chiral index $(n, m)$ of SWNTs to investigate the $(n, m)$ parametric space. The smallest stable endohedral structures were zigzag $(7,0)$ and chiral $(5,3)$ tubes having the diameter $5.48 \AA$ with an internal space still available for doping $\mathrm{Co}_{13}$ cluster 4.67 $\AA$ in size in different orientations.

When local spin density approximation (LDA functional) was applied, the zigzag $(7,0)$ nanotube with an across oriented $\mathrm{Co}_{13}$ cluster showed the magnetic moment 1.54 $\mu_{B}$ that is 4.8 times less than for along oriented $\mathrm{Co}_{13}$ cluster with the corresponding magnetic rate $7.34 \mu_{\mathrm{B}}$. In the case of generalized gradient approximation (GGA functional), the values were 2.99 and $8.14 \mu_{\mathrm{B}}$ in across and along orientations, respectively (Figure 1).

The similar pattern of magnetic activities depending on orientation of the $\mathrm{Co}_{13}$ cluster was found for the chiral $(5,3)$ tube with the same diameter $5.48 \AA$ as the zigzag $(7,0)$ tube. In the frame of LDA approach, when the $\mathrm{Co}_{13}$ cluster took a cross orientation to the channel, the magnetic rate was $3.42 \mu_{\mathrm{B}}$, and when an along orientation of $\mathrm{Co}_{13}$ cluster was considered, then the magnetic moment of the complex was $6.24 \mu_{\mathrm{B}}$ that is 1.8 times higher. When I used GGA method, the magnetism was estimated as 3.24 and $5.27 \mu_{\mathrm{B}}$, respectively. It is interesting that the difference in magnetic moments depending on $\mathrm{Co}_{13}$ cluster orientation was smaller for a chiral tube than for a zigzag tube with the same diameter.

An unexpected result was obtained from experiments on the chiral $(6,2)$ tube with a bigger diameter $5.65 \AA$. The magnetic moments of this filled nanotube for various orientations of $\mathrm{Co}_{13}$ cluster were smaller than in the case of the chiral $(5,3)$ tube, such as $1.15 \mu_{\mathrm{B}}$ for across orien-

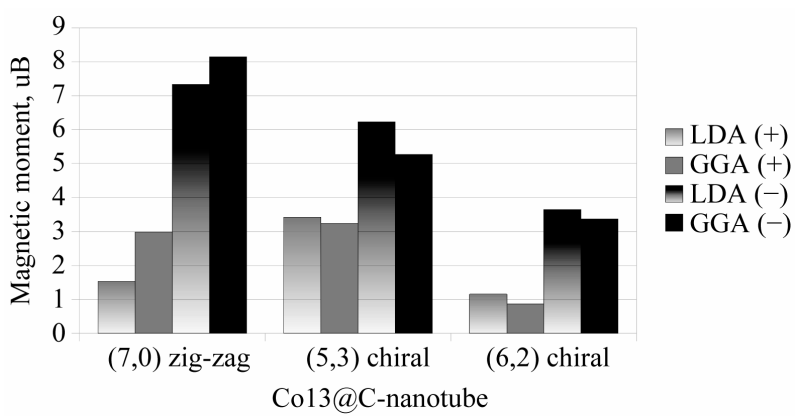

Figure 1. Total spin magnetic moments $S\left(\mu_{\mathrm{B}}\right)$ of endohedral carbon nanotubes depending on the chiral symmetry and position of $\mathrm{Co}_{13}$ impurity, where $(+)$ is across orientation, and (-) is longitudinal orientation of the $\mathrm{Co}_{13}$ cluster to the nanotube's channel.

tation and $3.65 \mu_{\mathrm{B}}$ for longitudinal orientation with LDA calculations, as well as 0.86 and $3.37 \mu_{\mathrm{B}}$, respectively for GGA technique.

In general, the correlation between LDA-CA and GGAPBE methods on the given experimental set was significant $(R=0.937)$.

\section{Analysis and Discussion}

As follows from the outcome above, the $\mathrm{Co}_{13}$ cluster emits more magnetism in an along orientation than in a cross orientation to the channel of a nanotube (Figure 1). This data is in good agreement with the result on doped by $\mathrm{Co}_{5}$ elongated fullerene $\mathrm{C}_{70}-D_{5 h}$ and confirms the earlier conclusion: the closer carbon and cobalt atoms, the less magnetic moment of the Co-C complex [13,14]. Nevertheless, this simple rule does not work in the matter of chiral nanotubes. As such, the $\mathrm{Co}_{13}$ cluster emits less magnetism in the $(6,2)$ tube with the diameter $5.65 \AA$ than in the $(5,3)$ tube with the diameter $5.48 \AA$, which demonstrates a nontrivial role of the carbon structure in magnetisation of Co-C complex. This finding is very interesting because the transmission electron microscopy, electron diffraction, and X-ray spectroscopy showed a structural transition of Co particles inside CNTs from hexagonal-close-packed (hcp) arrangement to face-centered-cubic (fcc) organization as a result of interaction between Co nanoparticles with CNTs [30].

Although the magnetic features of $\mathrm{Co}_{13}$ cluster in nanotubes were noticeable in our calculations, the hidden mechanisms of magnetism and peculiarity of electron actions in filled nanotubes are out of DFT approach [31, 32]. The chemical structure of nanotubes is composed of $\mathrm{sp}^{2}$ bonds, similar to those of graphite. Like in graphite, electrons can move along a tube, parallel to the graphene sheet. However, some tubes have a band gap like semiconductors that depends on chirality. For instance, $(n, n)$ armchair tubes are metals, chiral $(n, m)$ tubes with $n-m$ divisible by 3 are small band gap semiconductors, all 
other tubes are large-band gap semiconductors [33]. Hence, experimental nanotubes with chiral indexes $(7,0),(5,3)$, $(6,2)$ are large-band gap semiconductors. Nonetheless, they demonstrated different properties of magnetism with introduced $\mathrm{Co}_{13}$ cluster, depending on the chiral symmetry that was not observed early on regarding endohedral fullerenes with chirality types $(1,1)$ and $(2,0)[14]$.

\section{Conclusion}

The total spin magnetic moment $\mathrm{S}$ of $\mathrm{Co}_{13}$ cluster with icosahedral symmetry decreased in the SWNT environment more significantly in the case of across orientation of the cluster than in the longitudinal orientation to a channel of nanotube. The magnetism did not monotonically descent with the decreasing diameter of filled carbon tube like endohedral fullerenes. Thus, open ends of carbon nanotubes are an important factor in the magnetic behavior of SWNTs doped with metallic cluster.

The parametric space in which the chiral vector $(n, m)$ presented the symmetry of a nanotube was partly investigated. The magnetic moment of $\mathrm{Co}_{13}$ cluster embedded into carbon nanotubes of different chirality $(n, m)$ varied in a wide range. The landscape of $M=f(n, m)$ was very ruffle and unpredictable. Most structures were hard to calculate with LDA and GGA functionals. However, I hope a protonation of nanotube's ends and new approaches will possibly allow to investigate extensive $(n, m)$ space.

In summary, the magnetic states of endohedral carbon nanotubes were strongly dependent on the orientation of Me-cluster within nanotube and on the fine atomic structure of carbon shell, i.e. on the specific arrangements between the metal and carbon atoms.

\section{Acknowledgements}

The author thanks Heinz Eikmeyer and anonymous referees for helpful suggestions.

\section{REFERENCES}

[1] J. L. Rodríguez-López, F. Aguilera-Granja, K. Michaelian and A. Vega, "Structure and Magnetism of Cobalt Clusters," Physical Review B, Vol. 67, No. 17, 2003, Article ID: 174413. doi:10.1103/PhysRevB.67.174413

[2] F. Lopez-Urias, E. Cruz-Silva, E. Munoz-Sandoval, M. Torrones and H. Terrones, "Magnetic Properties of Individual Carbon Clusters, Clusters inside Fullerenes and Graphitic Nanoribbons," Journal of Material Chemistry, Vol. 18, No. 13, 2008, pp. 1535-1541. doi:10.1039/b716752k

[3] S. Blügel and G. Bihlmayer, "Magnetism of Low-Dimensional Systems: Theory," In: H. Kronmüller and S. S. P. Parkin, Eds., Handbook of Magnetism and Advanced Magnetic Materials, John Wiley \& Sons Ltd., Chichester, 2006, pp. 598-640.
[4] S. Sahoo, "Ab Initio Study of Free and Deposited Transition Metal Clusters," Ph.D. Dissertation, Fakultät für Physik der Universität Duisburg-Essen, 2011.

http://duepublico.uni-duisburg-essen.de/servlets/Derivate Serlet/Derivate-28204/Dissertation-Sanjubala_Sahoo.pdf

[5] H. W. Kroto, J. R. Heath, S. C. O’Brien, R. F. Curl and R. E. Smalley, "C $\mathrm{C}_{60}$ : Buckminsterfulleren," Nature, Vol. 318, No. 6042, 1985, pp. 162-163. doi:10.1038/318162a0

[6] S. Iijima, "Helical Microtubules of Graphitic Carbon," $\mathrm{Na}$ ture, Vol. 354, No. 6348, 1991, pp. 56-58. doi: $10.1038 / 354056 \mathrm{a} 0$

[7] M. P. Johansson, J. Jusélius and D. Sundholm, "Sphere Currents of Buckminsterfullerene," Angewandte Chemie International Edition, Vol. 44, No. 12, 2005, pp. 1843-1846. doi:10.1002/anie.200462348

[8] X. Lu and Z. Chen, "Curved Pi-Conjugation, Aromaticity, and the Related Chemistry of Small Fullerenes $\left(\mathrm{C}_{60}\right)$ and Single-Walled Carbon Nanotubes," Chemical Reviews, Vol. 105, No. 10, 2005, pp. 3643-3696. doi:10.1021/cr030093d

[9] S. Hong and S. Myung, "Nanotube Electronics: A Flexible Approach to Mobility," Nature Nanotechnology, Vol. 2, No. 4, 2007, pp. 207-208. doi:10.1038/nnano.2007.89

[10] J.-C. Charlier, X. Blasé and S. Roche, "Electronic and Transport Properties of Nanotubes," Reviews of Modern Physics, Vol. 79, No. 2, 2007, pp. 677-732. doi:10.1103/RevModPhys.79.677

[11] T. Makarova and P. Fernando, "Carbon-Based Magnetism: An Overview of the Magnetism of Metal Free Carbon-Based Compounds and Materials," Elsevier, Oxford, 2006.

[12] J. Kolosnjaj, H. Szwarc and F. Moussa, "Toxicity Studies of Carbon Nanotubes," Advances in Experimental Medicine and Biology, Vol. 620, 2007, pp. 181-204. doi:10.1007/978-0-387-76713-0 14

[13] A. Kuznetsov, "From Carbides to $\mathrm{Co}_{5}$ and $\mathrm{Co}_{13}$ Metallofullerenes: First-Principles Study and Design," American Journal of Biomedical Engineering, Vol. 2, No. 1, 2012, pp. 32-38. doi:10.5923/j.ajbe. 20120201.05

[14] A. Kuznetsov, "Magnetic Properties of Endohedral Complexes $\mathrm{Co}_{5} @ \mathrm{C}_{\mathrm{n}}$ Depending upon the Size and Symmetry of Fullerenes as Well as Orientation of Cobalt Cluster," Computational Materials Science, Vol. 54, 2012, pp. 204207. doi:10.1016/j.commatsci.2011.09.034

[15] M. S. Dresselhaus, G. Dresselhaus and P. C. Eklund, "Science of Fullerenes and Carbon Nanotubes," Academic Press, San Diego, 1996.

[16] V. V. Ivanovskaya, C. Köhler and G. Seifert, "3d Metal Nanowires and Clusters inside Carbon Nanotubes: Structural, Electronic, and Magnetic Properties," Physical Review $B$, Vol. 75, No. 7, 2007, Article ID: 075410. doi:10.1103/PhysRevB.75.075410

[17] P. Susmita, C. Sayan, P. Manh-Huong, M. Pritish and S. Hariharan, "Carbon Nano Straws: Nanotubes Filled with Superparamagnetic Nanoparticles," Nanotechnology, Vol. 20, 2009, Article ID: 485604. doi: $10.1088 / 0957-4484 / 20 / 48 / 485604$

[18] S. Melchor and J. A. Dobado, "CoNTube: An Algorithm 
for Connecting Two Arbitrary Carbon Nanotubes," Journal of Chemical Information and Computing Science, Vol. 44, No. 5, 2004, pp. 1639- 1646. doi:10.1021/ci049857w

[19] CoNTub, http://www.ugr.es/local/gmdm/java/contub/contub.html

[20] N. Guex and M. C. Peitsch, "Swiss-Pdb Viewer: A Fast and Easy-to-Use PDB Viewer for Macintosh and PC," Protein Data Bank Quaterly Newsletter, Vol. 77, 1996, p. 7.

[21] P. Hohenberg and W. Kohn, "Inhomogeneous Electron Gas," Physical Review B, Vol. 136, No. 3B, 1964, pp. 864871. doi:10.1103/PhysRev.136.B864

[22] T. Ozaki, "Variationally Optimized Atomic Orbitals for Large-Scale Electronic Structures," Physical Review B, Vol. 67, No. 15, 2003, Article ID: 155108. doi:10.1103/PhysRevB.67.155108

[23] T. Ozaki and H. Kino, "Numerical Atomic Basis Orbitals from H to Kr," Physical Review B, Vol. 69, No. 19, 2004, Article ID: 195113. doi:10.1103/PhysRevB.69.195113

[24] T. Ozaki and H. Kino, "Efficient Projector Expansion for the ab Initio LCAO Method," Physical Review B, Vol. 72, No. 4, 2005, Article ID: 045121. doi:10.1103/PhysRevB.72.045121

[25] OpenMX, http://www.openmx-square.org/

[26] D. M. Ceperley and B. J. Alder, "Ground State of the Electron Gas by a Stochastic Method," Physical Review Letters, Vol. 45, No. 7, 1980, pp. 566-569.
doi:10.1103/PhysRevLett.45.566

[27] J. P. Perdew, K. Burke and M. Ernzerhof, "Generalized Gradient Approximation Made Simple," Physical Review Letters, Vol. 77, No. 18, 1996, pp. 3865-3868. doi:10.1103/PhysRevLett.77.3865

[28] S. Datta, M. Kabir, S. Ganguly, B. Sanyal, T. Saha-Dasgupta and A. Mookerjee, "Structure, Bonding, and Magnetism of Cobalt Clusters from First-Principles Calculations," Physical Review B, Vol. 76, No. 1, 2007, Article ID: 014429. doi:10.1103/PhysRevB.76.014429

[29] S. Blügel, "Magnetism at the Nanoscale (Lecture)," Spring College on Computational Nanoscience, Trieste, 2010.

[30] X. Ma, Y. Cai, N. Lun, Q. Ao, S. Li, F. Li and S. Wen, "Microstructural Features of Co-Filled Carbon Nanotubes," Material Letters, Vol. 57, No. 19, 2003, pp. 2879-2884. doi:10.1016/S0167-577X(02)01391-5

[31] C.-K. Yang, J. Zhao and J. P. Lu, "Magnetism of Transition-Metal/Carbon-Nanotube Hybrid Structures," Physical Review Letters, Vol. 90, No. 25, 2003, Article ID: 257203. doi:10.1103/PhysRevLett.90.257203

[32] V. A. Basiuk and S. Irle, "DFT Calculations on Fullerenes and Carbon Nanotubes," Research Signpost, Trivandrum, 2008.

[33] M. S. Dresselhaus, G. Dresselhaus and P. Avouris, "Carbon Nanotubes: Synthesis, Structure, Properties, and Applications," Springer, Berlin, 2001. doi:10.1007/3-540-39947-X 